\title{
MUJERES Y GÉNERO EN ITALIA: "EN EL LABERINTO DE LA RED”
}

\author{
Dolores Ramírez Almazán y Leonarda Trapassi \\ Universidad de Sevilla
}

\begin{abstract}
This essay offers, on the one hand, a series of general reflections upon the possibilities that Internet generates in research, compilation of texts and materials and teaching from the perspective of Gender Studies. On the other hand, some Italian web pages and internet addresses are also discussed, which can be interesting from the cultural, literary or linguistic point of view.
\end{abstract}

\section{EL TELAR Y EL LABERINTO}

En la actualidad muchas personas saben que ADA no es una sigla de significado oculto, sino un claro homenaje a Ada Augusta Lovelace (1815-1852), la mujer que hace ahora más de un siglo ideó los fundamentos de este conocido lenguaje de programación. Como muchas otras mujeres de su tiempo ha sido durante demasiados años una perfecta desconocida y sólo desde hace poco hemos podido saber que trabajó junto a Charles Babbage, "perfecto conocido", en la creación de la "máquina diferencial", una especie de calculadora considerada el antepasado directo de los actuales ordenadores. La colaboración en el proyecto de esta mujer, matemática y humanista, fue, como es habitual, "discreta", callada, velada, siendo de ello un claro testimonio el acrónimo "A. A. L." con el que únicamente le era permitido firmar las notas a las publicaciones de aquellos trabajos.

Pero nada de todo esto puede sorprender pues, como la de otras muchas pioneras del siglo XIX, la historia de Ada Lovelace es, una vez más, una parte de esta gran historia que espera para ser rescatada del olvido, una parte que espera paciente para incorporarse a nuestro pasado común, a ese "nuestro referente"1.

Recordar en este momento a Ada Lovelace (1815-1852) es no sólo recordar a una de nuestras científicas pioneras, es también recordar el hecho de que las mujeres hemos estado ahí siempre, invisibles, ocultas, calladas, en un plano subalterno, pero vivas y formando parte de nuestra historia, de nuestra cultura, de nuestra literatura y, por supuesto, de la tecnología, que es también nuestra desde los comienzos y que actualmente debe seguir siéndolo, de una manera especial, en femenino.

\footnotetext{
${ }^{1}$ Como lo define Anna Santoro, que con su antología quiere contribuir en la elaboración de la gran historia de los sujetos femeninos y, en último término, "dare il senso della storia alle donne che non sanno di possederla e che, finché non la conosceranno, avranno maggiore difficoltà ad essere autonome [...] per costruire la propria soggettività" A. Santoro, Il Novecento, Antologia di scrittrici italiane del primo ventennio, Roma, Bulzoni, 1997, p. 13 .
} 
Pero además, recordar a Ada es "re-tejer" de nuevo la metáfora del telar ${ }^{2}$, y es preciso recordarlo porque esta metáfora sigue siendo el centro de la reflexión de los estudios de género en todos los ámbitos del saber. Hoy, con las nuevas tecnologías, con las autopistas de la información, con los nuevos sistemas de comunicación y sobre todo con "la internet" (el femenino atribuido es intencionado en algunas escritoras) esta metáfora ofrece un nuevo ángulo desde el que analizar la situación de los estudios de género, de los estudios de mujeres, como un recurso fundamental de información y como todo un universo de interrelaciones y de creación de una nueva subjetividad (Dona Haraway, Rosi Braidotti, etc.).

Así, muchos de los términos que tienen que ver con el campo semántico del telar, (tejido, entretejer, redes, etc.) aparecen con frecuencia en la literatura feminista como un lugar lingüístico desde el que dar vida a metáforas, imágenes y símbolos. Es el caso de Sadie Plant ${ }^{3}$, para quien el telar es una de las invenciones más claramente atribuibles a la mujer y quien, al mismo tiempo, recuerda que los primeros ordenadores, de la mano de Ada Lovelace, se concibieron precisamente a partir del modelo del telar de Jacquard. Y otra vez el nexo entre Penélope (el mito de tejer conocimiento) y las nuevas tecnologías. Y lo que es más importante, Plant atribuye al lenguaje informático nacido del telar el mérito de haber abierto nuevas fronteras al pensamiento, liberándolo de la estructura textual y jerárquica en la que se encontraba encasillado para abrirlo a una amplia estructura reticular y horizontal.

Hace tan sólo diez años una especie de consigna imperaba en toda la literatura que relacionaba internet (no la internet) con los estudios de género: la necesidad de no quedar al margen de la "red de redes", de "no perder el tren de las nuevas tecnologías", de no desaprovechar la oportunidad de alcanzar el mundo de la información, un espacio del que éramos y somos todavía en gran manera ignoradas, pero que podemos construir con otra mirada ${ }^{4}$. Hoy "La Internet", como le gusta llamarla a Montserrat Boix ${ }^{5}$, ha roto fronteras y ha permitido "que muchas mujeres de todo el mundo, interesadas en temas de género, puedan intercambiar experiencias, apoyos y estrategias, en definitiva, ha favorecido la Inter-relación." De ahí la importancia de crear redes de comunicación que "multiplican el trabajo de todas" y favorecen la construcción de espacios con perspectivas de género. Sin embargo, ésta sigue siendo una vindicación totalmente vigente.

\footnotetext{
${ }^{2}$ Metáfora recurrente en los estudios de género y en los estudios de literatura escrita por mujeres, desde el mitológico tejido de Penélope como símbolo de conocimiento, hasta, por citar otro más próximo en el tiempo y el espacio, el objeto de estudio del Seminario Internacional de la Asociación AUDEM, Sevilla, 2002 "Entretejiendo saberes" (Arriaga, M., et al. 2002).

3 "The future looms: weaving women and cybernetics", en S. Plant, Cybersexualities. A Reader on Feminist Theory, Cyborg and Cyberspace, Edinburg University Press, 1999, pp. 99- 118

${ }^{4}$ La situación en los estudios de Ingeniería Informática no es muy distinta a los de otros ámbitos de conocimiento y estudio: la presencia femenina es muy alta en los trabajos menos cualificados y más repetitivos, como insertar datos y cadenas de montaje. El antiguo prejuicio de la incompetencia técnica de las mujeres ha limitado nuestra presencia en los cargos donde se deciden proyectos y técnicas; Vid. S. CHIARANTINI, "Donne e computer: il genere della tecnologia", Internet. 21/10/04. <http://users.libero.it/scarmela/immagini/abstract.pdf $>$ (21-10-04).

${ }^{5}$ Vid. M. Boix, C. Fraga, V. Sedon, El viaje de las internautas: una mirada de género a las nuevas tecnologías,

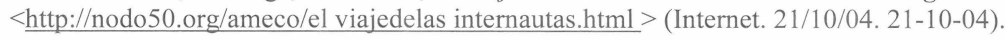


Si bien la presencia de las mujeres empieza a hacerse visible en la red primero en el ámbito anglosajón a partir de los 70, y unos años después, más o menos al mismo tiempo en distintos países de Europa, hay que esperar a la década de los 90 para asistir a una verdadera explosión de la presencia femenina en internet, así como al nacimiento, más que necesario, de las primeras redes electrónicas de mujeres. Han sido fundamentalmente académicas y periodistas las que con, por ejemplo, Pénélopes, Mujeresenred y GopherDonna en Francia, España e Italia respectivamente, han dado los primeros pasos hacia esa labor de interrelación, recopilación, coordinación, divulgación de proyectos e iniciativas personales, asociativos e institucionales, en el inmenso océano de la información. De este modo, gracias a estos grandes nodos de interrelación, es posible vislumbrar, si quiera mínimamente, lo que las mujeres escriben, piensan, hacen, transmiten por y para las mujeres desde la perspectiva de género.

En el caso concreto de Italia, en 1996 el Centro di Documentazione della Donna y la Asociación de Mujeres Orlando de Bolonia crean el servidor con contenidos de género Server Donne (www.women.it). De esta época es también Info@perla, como suplemento exclusivamente dedicado a las relaciones de las mujeres e internet de la mayor, más antigua y más amplia, Madreperla y que dará lugar al grupo Medea. Como en este caso, muchas revistas feministas de años anteriores y larga trayectoria terminan dando el salto a la versión digital. Así sucede con Il paese delle donne, (www.womenews.net), una de las más reconocidas, y con otras muchas más que han logrado colocarnos verdaderamente "en red", no sólo "en Internet", para unir la creatividad individual en un proyecto conjunto de información que es capaz de interpretar, de contar el mundo desde un punto de vista sexuado, demostrando como ya ha señalado Isabel de Torres ${ }^{6}$ que los Estudios de Género "representan una de las más significativas renovaciones que se han incorporado a la investigación en las últimas décadas".

Desde la perspectiva no sólo histórica o documental, sino, más en concreto, desde la perspectiva didáctica, y desde nuestra posición como docentes e investigadoras, hacerse una idea de cómo funciona Internet y hacer un buen uso de "La Internet" para que no se convierta en un interminable laberinto puede resultar bastante complicado. No bastan los buscadores para conseguir la información que nos interesa. Es más, puede causar verdadero pánico obtener alrededor de 300.000 páginas como resultado de la búsqueda con la palabra clave "estudios de género". No obstante, es de gran ayuda comprender que esa cantidad no es más que el cómputo de una información heterogénea indexada bajo aquella palabra clave, una especie de "sobredosis de información" (Montserrat Boix) "letal" cuando nos aventuramos a atravesar este inmenso océano sin conocer su funcionamiento, sin desarrollar las estrategias necesarias de búsqueda ${ }^{7}$ o, sobre todo, sin ni siquiera descartar aquellas páginas empeñadas en reproducir incansablemente el estereotipo de mujer bajo el modelo patriarcal, "reina y señora del hogar". En este sentido, si bien la

\footnotetext{
${ }^{6}$ I. De Torres Ramírez, Los recursos informativos documentales para los Estudios de las mujeres. Panorámica breve desde Europa. Universidad de Granada. Instituto de Estudios de la Mujer, p. 2, $<$ http://www.nodo50.org/mujeresred/isabeldetorres.html > (Internet. 21-10-04).

${ }^{7}$ Puede servir de ayuda para los que parten de cero o conocen sólo un poco el funcionamiento de internet, además del ya mencionado El viaje de las internautas, el texto de M. Calvo/ G. Rongaglia/ F. Ciotti/ M. A. Zela, Internet 2004, en particular el capítulo "Come si fa ricerca in Internet". El texto puede descargarse en

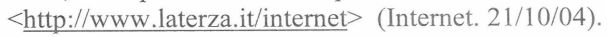


navegación por internet en ocasiones resulta ciertamente desorientadora, debe tenerse en cuenta la posibilidad de ofrecer a las mujeres en general (y a las alumnas y alumnos que se inician en los estudios de género o de literatura escrita por mujeres, en nuestro contexto particular), los instrumentos necesarios para acceder a la información, organizarla y valorarla.

\section{RED Y COMUNICACIÓN}

A la hora de reflexionar sobre los materiales existentes en la red, se deben tener en cuenta varios factores. Dado que en la práctica diaria de la enseñanza nos encontramos con el uso de forma acrítica del material existente en la red (es decir, sin discriminar o valorar la calidad o la procedencia de este material), resulta necesario un uso guiado y algunos consejos crítico-bibliográficos ante la avalancha de páginas existentes en la red, como parte de la introducción a los estudios de mujeres y estudios de género, (utilizando las definiciones italianas: "studi femminili e studi di genere").

El tipo de materiales que se señalan a continuación pertenecen al área italiana, y son resultado de una selección previa que pretende, teniendo en cuenta las distintas perspectivas de género, ofrecer una mirada crítica de la cultura italiana. En todo caso, se trata de documentos y materiales procedentes de universidades, grupos de investigación italianos y algunos extranjeros, siempre en lengua italiana.

Por otra parte y dado que la mayoría de las páginas que se crean y luego se abandonan ofrecen un interés relativo, el que sean páginas en continua actualización ha sido uno de nuestros criterios a la hora de seleccionar el material. La ventaja de la red es, efectivamente, su posibilidad de una continua expansión y, eventualmente, de la interacción y el diálogo.

La tipología de los materiales que se encuentran en la red es, obviamente, parecida a la existente en otras áreas culturales. En nuestra búsqueda y selección hemos podido comprobar que, análogamente a lo que sucede en otros ámbitos de conocimiento, la red, como el medio de comunicación más rápido y económico, amplifica las posibilidades en positivo y en negativo; es decir, en el ámbito de los estudios de género, gracias a la red, la comunicación y el intercambio cognoscitivo son más eficaces, pero, como contrapartida, es a través de la red como más rápidamente se transmiten las imágenes y clichés que reproducen el estereotipo del "angelo del focolare", de la mujer dedicada a estar a la última en moda y en cosmética.

Pero nuestra búsqueda se plantea claramente unos objetivos bien distintos. Lo que interesa aquí es sugerir una mirada crítica y un uso diferente de internet, como medio de trabajo en la docencia cotidiana, en especial en la perspectiva de la filología italiana (pero también de otras filologías, y sobre todo, evidentemente desde una perspectiva extranjera, dada la facilidad de acceso a la red). La red, en definitiva, puede ser un instrumento útil para diferentes operaciones como, en nuestro caso particular, a la hora de:

- recopilar textos literarios, ensayísticos y artículos;

- orientar entre las diferentes opciones de trabajo en los estudios de mujeres y de género;

- adquirir léxico específico;

- buscar bibliografía; 
- obtener información en torno a la historia de las mujeres y de los movimientos de reivindicación de los derechos de la mujer;

- buscar información sobre el desarrollo actual y grupos de investigación que trabajan en temas relacionados con la mujer, la construcción cultural del género y sus implicaciones socio-culturales en Italia;

- comparar distintas realidades nacionales;

- fomentar el diálogo y el intercambio de ideas.

Por lo que se refiere a los ejemplos prácticos de un posible uso de los materiales encontrados, aquí nos limitamos simplemente a comentar brevemente algunos sitios que pensamos pueden servir como base de diálogo, material de trabajo e investigación a través de internet .

Entre las numerosas revistas on-line DeA. Donne e altri (www.donnealtri.it) ofrece una mirada crítica a la prensa diaria y otras publicaciones. Es una revista en red creada por periodistas convencidas de que, a pesar de los numerosos cambios en la manera de hacer información, la prensa sigue mirando a un lector-usuario aparentemente neutro, pero en realidad de sexo masculino. Este mecanismo produce normalmente una selección de las noticias y un panorama informativo dedicado principalmente a un lector masculino. Como afirman las autoras y los autores de la revista en la presentación, "lo femenino irrumpe en la escena mediática cuando se trata de eventos que rompen las certezas radicadas". Así lo que se propone no es una discriminación al revés. Lejos de toda manipulación o forma de fundamentalismo, las y los periodistas de $D e A$ proponen otra mirada a la información que incluye también la narración de la normalidad de la vida de las mujeres y del cambio de la relación entre los sexos, tanto en la sociedad italiana como en otras sociedades. Este tipo de revistas puede ser de utilidad, sobre todo fuera de Italia, para, por ejemplo, contrastar y mirar de forma crítica la opinión de un periódico tradicional sobre la realidad italiana.

Resulta también interesante consultar páginas de centros de documentación, como Un portale di genere. Server donne (www.women.it). Se trata de la página de la asociación Orlando (en referencia a la conocida novela de Virginia Woolf) nacida en los años 70 en Bolonia, formada por mujeres italianas y extranjeras activas en el ámbito de la cultura, con el objeto de fomentar redes de relaciones e intercambios a nivel nacional e internacional. Además, a esta asociación se debe la creación en Bolonia del Centro di Documentazione delle Donne y la Biblioteca italiana delle Donne, como centros públicos y referencia necesaria para quienes se ocupan de la problemática de género en Italia. Server donne ofrece la sección "banche dati" que alberga enlaces con textos y catálogos on-line de la Biblioteca italiana delle Donne, así como el enlace con el Centro di Documentazione delle Donne.

Por otra parte, en la sección "risorse" se da acceso, bajo "i luoghi delle donne in Italia", a un listado de los centros de estudio de la mujer y estudios de género en las diferentes regiones de Italia.

No hay todavía muchas recopilaciones de textos de mujeres y sobre mujeres. Pero, por lo que se refiere a textos bio-bibliográficos sobre mujeres escritoras, artistas y críticas se puede citar la página de la asociación L'Araba Felice (www.arabafelice.it). Es una página importante, sobre todo, porque ofrece un diccionario bio-bibliográfico interactivo de las mujeres pertenecientes al ámbito de la creación y el conocimiento. No todas las entradas 
presentan la misma calidad, pero ofrece una cierta orientación a la hora de iniciar la investigación sobre una escritora o una artista gracias a las reseñas y artículos que se pueden descargar.

Entre las páginas de centros de investigación universitarios y no universitarios destaca la página del Seminario interdisciplinare di studi di genere. Università Federico II, de Nápoles (www.storia.unina.it/donne/index.html). Es un sitio interesante sobre todo por los hipertextos a los que se puede acceder. Así, por ejemplo, desde "Il Risorgimento invisibile" se puede obtener un diccionario, una serie de perfiles bio-bibliográficos e información sobre figuras femeninas del "Risorgimento". Se podría añadir también Donne e conoscenza storica (www.url.it/donnestoria). La página, constituida en el año 1989, está gestionada por un grupo de profesoras la "Comunità di Pratica e riflessione pedagogica e di ricerca storica" y ofrece también hipertextos, textos, artículos, reseñas y sugerencias bibliográficas sobre temas de historia, cultura y literatura relacionados con las mujeres.

Muchísimas son sin embargo las páginas de asociaciones. Destacamos la página de la Casa Internazionale delle Donne (www.casainternazionaledelledonne.org) de la federación de asociaciones del movimiento de igualdad de oportunidades de Roma. Como tal es relativamente reciente (2002) si bien se remonta a proyectos anteriores, y tiene su sede en la antigua cárcel de mujeres "Ospizio della Santa Croce", edificio histórico perteneciente a la Iglesia desde 1615 que en la actualidad, tras una larga serie de episodios de restauración, paradójicamente se ha convertido en una estructura abierta y bien distinta: laboratorio, centro de documentación y de organización de seminarios y otros eventos, asesoría jurídica, para la salud y psicológica, etc.

Además de información sobre actividades y eventos de la asociación y de otras organizaciones ofrece, en la sección "letture", reseñas y fichas bibliográficas de publicaciones recientes relacionadas con la escritura femenina, la historia de las mujeres, los estudios de género, etc.

También hemos consultado la página de la Associazione per una Libera Università delle Donne (www.universitadelledonne.it). Se trata de una organización nacida en Milán de un grupo autofinanciado, de mujeres activas en el campo de los cursos para adultos y los consultorios para la salud de la mujer. Fundamentalmente se ha ocupado de temas feministas y se ha abierto progresivamente a nuevas áreas de reflexión (cibertecnologías, guerra, encuentro con mujeres de otros países y culturas, etc.). Las actividades consisten en cursos, seminarios, grupos de trabajo, etc. En las secciones "libri", "film" y "storia" se pueden leer reseñas, fichas y artículos sobre literatura, ensayos, películas, de y sobre mujeres y sobre temas relacionados con los estudios de género, que pueden ser un útil material de base para trabajar e investigar.

Entre las asociaciones que a través de internet ofrecen informaciones sobre actividades de investigación, señalamos también la Società italiana delle letterate (SIL) (www.societadelleletterate.it). Nacida en el año 1996, por iniciativa de un grupo de mujeres dedicadas a la investigación y a la práctica de la escritura y de la literatura, ofrece en su página web información sobre diversas actividades, seminarios y congresos.

Es productivo también navegar a través de las páginas institucionales. El Sito sulle politiche di genere del Comune di Torino (www.comune.torino/it/politichedigenere), ofrece por ejemplo abundante información en el campo de las políticas de género, que se refieren 
al ámbito local y, en general, a toda Italia, al tiempo que acoge el interesante proyecto de investigación "Quante donne puoi diventare", financiado por la Regione Piemonte, que puede servir de modelo en reflexiones análogas. El proyecto se propone el análisis crítico de libros ilustrados y de libros de textos para niñas y niños en edad escolar, entendidos como el principal vehículo de transmisión de modelos comunes de mujer y hombre socialmente aceptados, con la finalidad de elaborar un método susceptible de ser utilizado por maestros, bibliotecarios y padres a la hora de descodificar las imágenes simbólicas de la familia y de la sociedad actual contenidas en estas primeras lecturas. El sitio da acceso a información y bibliografía sobre el tema.

Pasando a las editoriales y librerías especializadas, mencionamos aquí sólo la página de la Libreria delle donne: (www.libreriadelledonne.it). Como se sabe, la "Libreria delle donne" de Milán no es una simple librería. Existe desde 1975, desde una de las épocas más intensas de las luchas feministas y se autodefine como "realtà politica composita e in movimento", "luogo storico della pratica di relazione"; edita publicaciones y una revista trimestral ("Via Dogana", nombre de la antigua sede), y organiza discusiones políticas, presentaciones de libros y otras muchas actividades. Esta página resulta útil porque permite la interacción, la participación en numerosos foros, debates e intercambio de opiniones y también porque permite descargar (en la sección "cosa c'è di nuovo") una selección de artículos de actualidad de varios periódicos, relacionados con temáticas de género. La sección "libri preziosi" además propone una bibliografía mínima, pero comentada, de obras literarias y ensayísticas fundamentales, no sólo italianas, en los estudios de género. La sección "mappe monde", título que de manera provocativa transforma en femenino plural la palabra "mondo", permite relacionarse con asociaciones, otras iniciativas interesantes y con editoriales extranjeras e italianas. En varias de las secciones que componen el sitio existe la posibilidad también de enviar materiales y textos.

Resultan de gran utilidad también las páginas de proyectos específicos, como Medea. Comunicazione digitale. L'altra metà della rete: (www.provincia.venezia.it/medea/index.shtml). Es un sitio telemático nacido en 1997, creado por un grupo de mujeres periodistas y especialistas en teoría de la comunicación. Parte de varias experiencias anteriores y tiene como objetivo profundizar a través de la perspectiva de género en la problemática relacionada con la sociedad digital. También pretende "dar voz" a aquellas mujeres cuya experiencia los medios de comunicación convencionales no tienen en cuenta. Uno de los proyectos a los que se dedica en la actualidad es, por ejemplo, la documentación sobre las mujeres albanesas que viven en Italia con entrevistas y reportajes. La página contiene, además, un archivo con los números anteriores de las revistas Infoperla y Madreperla, hasta 1999, con contribuciones sobre el espacio de la comunicación de las mujeres. La sección "eventi", constantemente actualizada, señala varias iniciativas y manifestaciones y alberga revistas, artículos y otros textos dedicados a libros, exposiciones, etc. con temáticas de género.

De entre todos estos comentarios, lo que en realidad interesa poner de relieve aquí es, fundamentalmente, el hecho de que, a través de un trabajo de búsqueda y selección y una navegación guiada por criterios y objetivos determinados, se puede profundizar y suscitar 
interés por las principales temáticas de género a varios niveles ${ }^{8}$, interactuando, dialogando y elaborando textos destinados a los distintos foros de discusión, y contribuyendo, de este modo, al establecimiento de una verdadera red de comunicación y reflexión en torno a nuevas temáticas en la investigación científica. En este caso, la red, en cuanto archivo potencialmente ilimitado, puede cubrir las necesidades en una primera toma de contacto y en el momento inicial de recopilación de ideas y materiales que preparan la investigación en el sentido tradicional. Y, por supuesto, la red es el lugar idóneo para la difusión de toda investigación.

Retomando las reflexiones generales sobre tecnología y género se puede considerar, en definitiva, que la red representa un espacio de diálogo y comunicación, y, obviamente, un espacio "con perspectiva de género" también en el mundo académico.

En todo caso, no podemos olvidar que el dominio del punto de vista masculino ${ }^{9}$ en un sector concreto no se basa sólo y exclusivamente en factores numéricos, del mismo modo que la paridad total no consiste sólo en aumentar el número de mujeres que participan en el desarrollo tecnológico. Por ello, sólo olvidando la tendencia a la uniformidad con la cultura dominante, se pueden hacer valer y valorar la subjetividad de las mujeres y otros puntos de vista, y será posible producir una tecnología realmente nueva y distinta. Es importante ser conscientes de que la perspectiva de género puede contribuir en la creación de nuevos universos de significación o nuevos órdenes simbólicos en los que la tecnología no se limite a ser un mero instrumento de poder, sino una herramienta útil para mejorar las relaciones humanas y la calidad de la vida. Pero además, debemos insistir con Cristina Fraga, en la necesidad de la creación de verdaderas redes de comunicación ${ }^{10}$ que "multiplican el trabajo de todas y fortalecen el movimiento internacional de las mujeres y la construcción de espacios con perspectiva de género"11 (Boix/Frege/Sedon 2004: 4).

\section{REFERENCIAS BIBLIOGRÁFICAS}

ARRIAGA, M. et al., Entretejiendo saberes. Actas de IV Seminario de la Asociación Universitaria de Estudios de Mujeres (AUDEM), Universidad de Sevilla, 2002 (publicación en CD-Rom).

\footnotetext{
${ }^{8}$ Por ejemplo utilizando este material también en una clase de lengua, de expresión oral o escrita en italiano, asignando varias tareas que puedan guiar la navegación en Internet para la presentación y la discusión en clase, o a la hora de la búsqueda bibliográfica para la investigación en un nivel superior, como en los cursos de doctorado.

9 Si atendemos a Silvia Chiarantini (2004) el saber tecnológico parece haberse construido sobre la imagen y el imaginario masculino proponiendo estas disciplinas como objetivas, desvinculadas de los valores humanos y sociales. La imagen más difundida de la tecnología nos lleva a una forma de racionalidad sin error, a la obsesión por el objeto en si mismo, al pragmatismo. Rasgos todos ellos muy lejanos de la tradicional identidad femenina que valora las relaciones, la fantasía, la creatividad y la cooperación.

${ }^{10}$ Con ocasión del V Congreso Internacional de la Asociación AUDEM, Sevilla, octubre 2005 planteamos la conveniencia de crear el nodo de conexión e interrelación de los estudios de género en todas las disciplinas en el ámbito de las Universidades Españolas, considerando que AUDEM es una adecuada plataforma desde la que implantar esa matrix inexistente.
} 
BOIX, M./FRAGA, C./SEDON, V., El viaje de las internautas: una mirada de género a las nuevas tecnologías, Ameco. Asociación española de Mujeres Profesionales de Medios de Comunicación Internet. 21-10-04, $<$ http:www.nodo50.org/ameco/el viaje de las internautas.html $>$.

CALVO, M.,/ RONCAGLiA, G. / CIOTTI, F./ ZELA, A. M., Internet 2004, Bari, Laterza, 2004; también en versión electrónica: Laterza. Internet. 21-10-2004. $<$ http://www.laterza/internet $>$.

CHIARANTINI, S., "Donne e computer: il genere della tecnologia", Internet. 21-10-04. $<\underline{\text { http://www. users.libero.it/scarmela/immagini/abstract.pdf }}>$.

DE TORRES RAMÍREZ, I., "Los recursos informativos documentales para los Estudios de las mujeres. Panorámica breve desde Europa", Ameco. Asociación española de Mujeres Profesionales de Medios de Comunicación. Internet. 21-10-2004, $<$ http://www.nodo50.org/mujeresred/isabel de torres.htlm $>$.

COMETA, M., (Ed.), Dizionario degli studi culturali, Roma, Meltemi, 2004.

GNISCI A., (Ed.), Introduzione alla letteratura comparata, Milano, Bruno Mondadori, 1999.

LANFRANCO, M., (Ed.), Il web delle donne. Guida ai siti femminili in internet, Genova, Erga edizioni, 2002.

PLANT, S., Cybersexualties. A Reader on Feminist Theory, Cyborg and Cyberspace, Edinburg, University Press. 1999.

SANTORO, A., Il Novecento. Antologia di scrittrici italiane del primo ventennio, Roma, Bulzoni, 1997.

SPAGNOLETTI, R., I movimenti femministi in Italia, Roma, Savelli, 1978.

VV.AA., Los estudios de mujeres hacia el espacio común europeo, Sevilla, Arcibel, 2005.

\section{Sitios WEB COMENTADOS:}

Casa Internazionale delle Donne: $<$ http://www.casainternazionaledelledonne.org $>$.

Dea. Donne e altri: $<$ http://www.donnealtri.it $>$.

Donne e Conoscenza storica: $<\underline{\mathrm{http}} / / / \mathrm{www}$.urlit/donnestoria $>$.

IL paese delle donne on line: $<$ http://www.womenews.net $>$.

L'Araba Felice: $<$ http://www.arabafelice.it $>$.

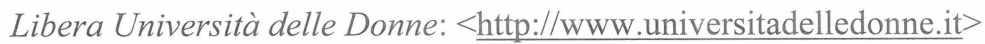

Libreria delle donne: $<$ http://www.libreriadelledonne.it $>$

Medea. Comunicazione digitale. L'altra metà della rete:

$<$ http://www.provincia.venezia.it/medea/index.shtml $>$.

Seminario interdisciplinare di studi di genere. Università Federico II:

$<$ http://www.storia.unina.it/donne/index.html $>$.

Sito sulle politiche di genere del Comune di Torino

$<$ http://www.comune.torino/it/politichedigenere $>$.

Società italiana delle letterate $(S I L):<\underline{\mathrm{http}}$ ://www.societadelleletterate.it $>$ 
Un portale di genere. Server donne: $\langle$ http://www.women.it $>$. 